\title{
Preexposure to situational cues produces a direct relationship between two-way avoidance learning and shock intensity
}

\author{
WALLACE R. McALLISTER, DOROTHY E. McALLISTER \\ STEPHEN E. DIETER, and JAMES H. JAMES \\ Northern Illinois University, DeKalb, Illinois 60115
}

\begin{abstract}
In Experiment 1, four groups of subjects ( $n=16$ each) were exposed to the situational stimuli of a shuttlebox apparatus for $4 \mathrm{~h}$. Subsequently, 200 two-way avoidance trials were administered (100/day) with either .3- or 1.6-mA shock and with either small or large reward (presence or absence of visual stimuli following the response). Avoidance performance was directly related to shock intensity on both days and to magnitude of reward on the 2nd day. In Experiment 2, four groups of subjects ( $\mathrm{n}=24$ each) were given $4 \mathrm{~h}$ of exposure either to the situational stimuli of the shuttlebox or to a neutral box. Then, 10 two-way avoidance trials were given with $1.6-\mathrm{mA}$ shock. Subsequently, subjects were allowed to escape from one of the shuttlebox compartments to an adjacent safe box. Following preexposure to situational stimuli, avoidance performance was superior whereas escape-from-fear performance was inferior. This latter finding demonstrated that less fear of situational cues was present during avoidance training in the preexposed condition. All of these results support the effective reinforcement theory, an extension of two-factor theory, which emphasizes the importance for avoidance learning of the amount of fear of situational cues present following a response.
\end{abstract}

In a one-way task in which subjects can avoid shock by moving to an area distinctively different from the one where shock is administered, avoidance performance has been found to be directly related to shock intensity (Dieter, 1976; Moyer \& Korn, 1966). However, when an avoidance response does not remove the subject from situational stimuli which have been paired with shock, as in signaled two-way or barpress avoidance tasks, performance has been found to be related inversely to shock intensity, at least within the range of intensities usually employed. This latter finding is, in fact, one of the most reliable in the avoidance learning literature (e.g., Anisman \& Waller, 1972; Bauer, 1972; Cicala \& Kremer, 1969; D'Amato \& Fazzaro, 1966; Freedman, Hennessy, \& Groner, 1974; Kurtz \& Shafer, 1967; Levine, 1966; McAllister, McAllister, \& Dieter, 1976; McAllister, McAllister, \& Douglass, 1971; Moyer \& Korn, 1964; Theios, Lynch, \& Lowe, 1966).

This research was supported in part by Grant BMS71-00845 from the National Science Foundation and Grant MH-29232 from the National Institute of Mental Health. A portion of these data was presented at the 1975 meeting of the Midwestern Psychological Association. The authors are indebted to Michael J. Grelle and Michael T. Scoles for aid in the collection and analysis of the data. Requests for reprints should be sent to Wallace R. McAllister, Psychology Department, Northern Illinois University, DeKalb, Illinois 60115. S. E. Dieter is now at Valparaiso University, St. Louis Divison, St. Louis, Missouri, and J. H. James is now at Yale University.
A theoretical position which seems to account for the above findings is the effective reinforcement theory proposed by McAllister et al. (1971, 1976) as an extension of the two-factor theory of avoidance learning (Mowrer, 1947). According to this position, the effective reinforcement for an avoidance response is related (a) positively to the amount of fear reduction occurring with the response and (b) negatively to the amount of fear present following the response. In addition, as the total amount of fear increases, it is assumed that the amount of fear present following the response (b) becomes relatively more important than the amount of fear reduction (a) in determining effective reinforcement. In the signaled twoway avoidance task, fear becomes conditioned to both the discrete conditioned stimulus (CS) and to the situational cues of each shuttle compartment when shock occurs on escape trials, with more fear being conditioned to these stimuli the more intense the shock. Although the amount of fear reduction would be greater in a strong- than in a weak-shock group when the CS is terminated following an avoidance response, this advantage is more than offset by the greater amount of fear of situational cues present following the response. Thus, with strong shock, there would be less effective reinforcement, and hence, avoidance performance would be inferior. In contrast, in a one-way avoidance task, where the response leads to a distinctive safe area, the amount of fear of situational cues following the 
response would be zero or minimal. Under this circumstance, effective reinforcement would be determined simply by the amount of fear reduction occurring with the response. Therefore, avoidance performance should be superior with strong shock.

Another way to state the present position is to say that effective reinforcement is a function of the amount of fear reduction relative to the total amount of fear. Phrased in this way, it is clear that the idea bears a close similarity to the notion of a relative reinforcement difference limen of shock intensity reported by Campbell (1956). He investigated the difference in shock intensities required to support a preference for the weaker of two shocks at different absolute levels of shock. With increases in shock levels, the maintenance of this preference was found to require an increase in both the absolute difference and the proportional difference between the two shock intensities. The implication of these findings for an instrumental learning task can be illustrated with the results of Campbell and Kraeling (1953). They found superior escape learning when rats were trained to run from a $300-\mathrm{V}$ to a $200-\mathrm{V}$ shock than from a $400-\mathrm{V}$ to a $300-\mathrm{V}$ shock. Thus, a constant amount of shock reduction was less effective as the absolute level of shock was increased. Furthermore, they found that escape was faster from a $200-\mathrm{V}$ to a $100-\mathrm{V}$ shock than from a $400-\mathrm{V}$ to a $200-\mathrm{V}$ shock. Under the latter condition, the absolute amount of shock reduction was greater than under the former condition, but the proportional amount of shock reduction was equal. These data support the notion that as the level of shock increases, both the absolute decrease in shock and the proportional decrease in shock must both be greater to provide equal reinforcement and, hence, equal performance. The effective reinforcement theory assumes these same relationships, but is concerned with fear reduction rather than shock reduction.

If the effective reinforcement theory as applied to the two-way task is correct, reducing the amount of fear of situational cues present following a response should give the strong-shock group a relative advantage over the weak-shock group, because this source of fear has a greater negative effect in determining reinforcement when shock is strong than when it is weak. The result would be to alter the magnitude of the usually obtained inverse relationship. Whether it would be reduced, eliminated, or reversed would depend on the extent to which effective reinforcement was increased in the strong-shock, relative to the weak-shock, group. The initial attempt to test this proposition (McAllister et al., 1976) used a large-reward condition in which CS termination was supplemented by the removal after the response of fear-eliciting visual situational cues. This manip- ulation facilitated performance in the weak-shock condition as compared to a group with CS termination alone (small reward) but had no effect when shock was strong. A likely reason for the failure to alter the usual inverse relationship as expected is that, with strong shock, fear of the tactual cues of the grid is of more importance than fear of the visual cues. Support for this contention is provided by Modaresi (1975), who found that when tactual cues of the grid, but not visual cues, were removed after a response, the usual inverse relationship was eliminated.

Because the above results indicate that fear conditioned to both the visual and tactual cues of the apparatus affect avoidance performance, a procedure which minimizes fear of both sources following the response might lead to a direct relationship between performance and shock intensity. One such procedure was employed by Dieter (1977). Prior to administering 65 two-way avoidance trials with either weak or strong shock, he exposed half of the subjects in each shock condition to the situational cues of the apparatus for 4 hours and the other half to a neutral box. Presumably, preexposure to the situational cues would result in a latent inhibition effect (e.g., Lubow, 1973; Rescorla, 1971) so that subsequent conditioning of fear to those cues on escape trials during avoidance training would be minimized. $\mathrm{He}$ found that without preexposure the typical inverse relationship occurred, but that with preexposure the weak- and strong-shock groups performed the same; that is, the inverse relationship was eliminated. The trends of the performance curves suggested that had additional avoidance trials been given, the strong-shock group may have eventually outperformed the weak-shock group, thus reversing the inverse relationship.

Experiment 1 had a dual purpose: (1) to determine whether extended two-way avoidance training following preexposure to the situational cues would lead to a direct relationship between shock intensity and avoidance performance, and (2) to investigate under the preexposure condition the effect of the reward manipulation described above (McAllister et al., 1976).

\section{EXPERIMENT 1}

\section{Method}

Apparatus. The shuttlebox, $444 \mathrm{~mm}$ long $\times 165 \mathrm{~mm}$ wide $\times$ $152 \mathrm{~mm}$ high, was divided into two identical compartments by a partition containing a black guillotine door, $57 \mathrm{~mm}$ wide $\times$ $89 \mathrm{~mm}$ high, which rested on a hurdle $63 \mathrm{~mm}$ high. The walls of each compartment were metal, painted white, and the floor was a grid consisting of 18 stainless steel rods, $3 \mathrm{~mm}$ in diameter, spaced $13 \mathrm{~mm}$ apart. Each floor was hinged to serve as a floor switch. Scrambled shock, provided by a Grason-Stadler shock generator, Model 700, was the UCS. Hinged to the top of each compartment was a box which contained two light sources and served as a cover. The bottom of each of these covers consisted 
of hardware cloth, $86 \mathrm{~mm}$ above which was a pane of ground glass. One light source provided illumination of $28 \mathrm{~lx}$; the other, the visual component of the compound CS, 1,029 lx. Together, the illumination from the two light sources measured $1,056 \mathrm{~lx}$. For the small-reward condition, the illumination of $28 \mathrm{~lx}$ was present in both compartments at all times. For the large-reward condition, the illumination of $28 \mathrm{~lx}$ was present only in the to-be-entered compartment at the beginning of each trial and was response terminated. Therefore, the increase in intensity of the visual stimulus which occurred with the onset of the CS in the compartment occupied by the subject and the decrease which occurred following a response were approximately equal for the two reward conditions $(1,029 \mathrm{~lx}$ for large reward and $1,028 \mathrm{~lx}$ for small reward). The white-noise component of the compound CS was provided by a Grason-Stadler white-noise generator, Model 901B, which increased the ambient noise level in the apparatus from 51 to $53 \mathrm{~dB}$. A very low level of room illumination $(2 \mathrm{~lx})$ was used throughout the experiment.

Subjects and Design. The subjects were 64 naive, female hooded rats, 114-120 days of age, from the colony maintained by the Psychology Department of Northern Illinois University. They were assigned at random to one of four groups of 16 subjects each. One additional subject was discarded for failure to escape shock within $60 \mathrm{sec}$ on 10 consecutive trials. No failures to escape occurred for any other subject. Except during the experimental treatments, the subjects were housed in pairs with food and water available ad lib.

For two groups of subjects, the shock intensity was $.3 \mathrm{~mA}$; for the other two, $1.6 \mathrm{~mA}$. The shock was discontinuous (.75 sec on and $2.00 \mathrm{sec}$ off). Within each shock-intensity condition, one group was given shuttlebox training with large reward (L) and the other with small reward (S). In the large-reward condition, because the illumination was terminated with a shuttle response, fear-eliciting visual situational cues were absent during the intertrial interval (ITI). In the small-reward condition, because the illumination was not terminated following a shuttle response, fear-eliciting visual situational cues were present during the ITI. The four groups were designated .3-S, .3-L, 1.6-S, and 1.6-L.

Procedure. On each of the first 4 days of the experiment, the subjects were handled in pairs for $5 \mathrm{~min}$, being alternately picked up and petted and placed on a table which they could explore. They were then placed in the apparatus, one in each compartment, with the guillotine door closed. The illumination in each compartment was $28 \mathrm{~lx}$. After 15, 30, and $45 \mathrm{~min}$, each subject was removed from one compartment and placed in the other. Thus, each subject was exposed for $30 \mathrm{~min}$ on each day to the cues of each compartment. Total exposure to the situational cues was, therefore, $4 \mathrm{~h}$.

On each of the next 2 days, 100 two-way avoidance trials were administered to each subject. Each trial began with the opening of the guillotine door and the simultaneous presentation of the compound CS. Shock was presented if the subject did not trip the floor switch in the opposite compartment within $5 \mathrm{sec}$ following CS onset. A reponse following shock onset terminated the CS and UCS (escape), whereas a response occurring during the 5-sec CS-UCS interval terminated the CS and prevented shock onset (avoidance). After a response, the subject remained in the compartment it had entered for a 30-sec ITI, after which the next trial was begun. If an escape response did not occur within $55 \mathrm{sec}$ following UCS onset, the guillotine door was closed, the CS and UCS were terminated, a latency of $60 \mathrm{sec}$ was recorded, and the next trial was begun $30 \mathrm{sec}$ later in the same compartment. Latencies were measured with a Hunter Klockounter in units of $.01 \mathrm{sec}$ from the opening of the guillotine door and CS onset to the depression of the floor in the opposite compartment. Approximately 23-25 h intervened between the daily experimental treatments.

\section{Results}

Percentage of avoidance responses. Figure 1 presents the mean percentage of avoidance responses

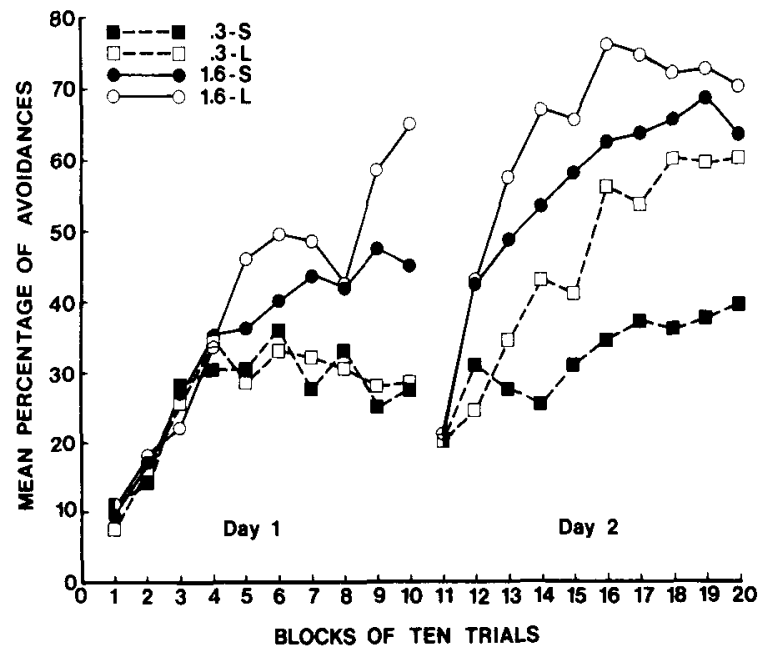

Figure 1. Mean percentage of avoidances on Days 1 and 2 of two-way avoidance training as a function of blocks of 10 trials for weak-shock $(.3 \mathrm{~mA})$ and strong-shock $(1.6 \mathrm{~mA})$ groups with small (S) and large (L) reward. All groups were preexposed to the situational cues.

in blocks of 10 trials for each group on Days 1 and 2 of two-way avoidance training. It is apparent that the superiority of performance of the strongshock groups which emerged on Day 1 was maintained on Day 2. Only on Day 2 was there a clear tendency at each shock level for the large-reward group to show better avoidance performance than the small-reward group. The decrement in performance from the last block of trials on Day 1 to the first block on Day 2 is not an uncommon phenomenon (e.g., Anderson, 1969; Freedman et al., 1974).

A repeated-measures ANOVA of the percentage of avoidance responses in blocks of 10 trials was computed separately for each day, with shock intensity and reward magnitude as the between-subjects factors and trial blocks as the within-subject factor. On Day 1, trial blocks $[\mathrm{F}(9,540)=22.18, \mathrm{p}<.001]$ and the interaction between trial blocks and shock intensity $[F(9,540)=5.06, p<.001]$ were significant. Because of the interaction, a factorial analysis was computed for each trial block. A significant effect of shock intensity was obtained on Trial Blocks 7, 9, and $10[\mathrm{~F}(1,60)=4.35,10.21,12.23 ; \mathrm{p}<.05,<.005$, $<.001$, respectively], reflecting the superiority of the strong-shock groups. No other effects were significant.

On Day 2, trial blocks $[\mathrm{F}(9,540)=26.15, \mathrm{p}<.001]$, shock intensity $[F(1,60)=7.39, p<.01]$, and the interaction of trial blocks with shock intensity $[F(9,540)=2.28, p<.02]$ and of trial blocks with reward magnitude $[\mathrm{F}(9,540)=1.89, \mathrm{p}=.05]$ were significant. These interactions with trial blocks reflect the more rapid improvement in performance over trials of the strong-shock as compared to the weak-shock groups and of the large-reward as compared to the small-reward groups. Because of the 
interactions, factorial analyses were computed for each trial block. The superior performance of the strong-shock over the weak-shock groups, indicated in the figure, was found to be reliable on Trial Blocks 13-19 (all ps $<.025$ ) and marginally reliable on Trial Blocks 12 and $20(p=.076$ and .060 , respectively). Despite the significant interaction between trial blocks and reward magnitude, the reward effect was found to be only marginally significant on Trial Blocks 16 and 18 ( $\mathrm{p}=.055$ and .087 , respectively). On no trial block did the interaction between shock intensity and reward magnitude approach significance. The increase in performance for each group on each avoidance training day shown in Figure 1 was found with Trial Blocks by Subjects analyses to be reliable in each instance (ps ranged from $<.025$ to $<.001)$.

Latency of avoidance responses. Evidence for the relevance of the reward-magnitude variable is provided by the avoidance latencies. As shown in the right portion of Figure 2, the mean avoidance latencies on Day 2 were lower at each shock level for the large-reward condition than for the small-reward condition. It is also clear from the figure that on both days the strong-shock groups avoided with shorter latencies than did the weak-shock groups. These data were analyzed with a repeated-measures ANOVA computed separately for each day of training. The between-subjects factors were shock intensity and reward magnitude, and the within-subject factor was blocks of 50 trials. Unweighted means ANOVAs were employed because some subjects did not make an avoidance response on both blocks of trials. The number of subjects in the groups ranged from 13 to 15 for these analyses.

On Day 1 , shock intensity $[F(1,52)=10.00, p<$
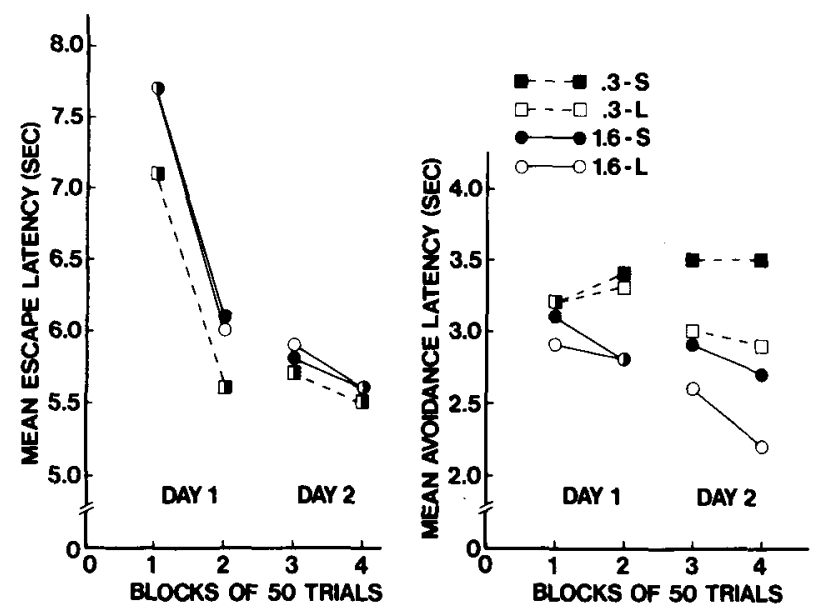

Figure 2. Mean escape latency (left panel) and mean avoidance latency (right panel) as a function of blocks of $\mathbf{5 0}$ trials on Days 1 and 2 of two-way avoidance training for weak-shock $(.3 \mathrm{~mA})$ and strong-shock (1.6 $\mathrm{mA})$ groups with small (S) and large (L) reward. All groups were preexposed to the situational cues.
$.005]$ and the interaction of shock intensity and trial blocks $[F(1,52)=4.31, p<.05]$ were significant. Analyses of the simple effects indicated that on the first trial block neither of the main effects nor the interaction was significant, whereas on the second trial block, shock intensity was significant $[F(1,52)$ $=14.81, p<.001]$. The changes in performance over trial blocks were not significant for either shock level.

On Day 2, shock intensity $[\mathrm{F}(1,52)=25.82, \mathrm{p}<$ $.001]$, reward magnitude $[F(1,52)=14.49, \mathrm{p}<.001]$, trial blocks $[F(1,52)=8.73, p<.005]$, and the interaction between trial blocks and shock intensity $[F(1,52)=5.19, \mathrm{p}<.05]$ were significant. Analyses of the simple effects indicated that on each trial block shock intensity was significant $[F(1,52)=14.38$, 29.39 , respectively; ps $<.001$ ], as was reward magnitude $[F(1,52)=8.62,15.56 ; p<.005,<.001$, respectively]. The decrease in latency over trial blocks was significant for the strong-shock $[F(1,27)=$ $13.73, \mathrm{p}<.001]$ but not for the weak-shock condition. None of the interactions approached significance.

Latency of escape responses. In the left portion of Figure 2, the mean escape latencies for the several groups are plotted in blocks of 50 trials. These data were analyzed in the same manner as the avoidance latencies except that an unweighted means analysis was required only on Day 2; one subject in each of the strong-shock groups made 50 avoidance responses on the last block of trials. The decrease in escape latencies indicated in the figure was significant on both Day $1[F(1,60)=369.77, p<.001]$ and Day 2 $[F(1,58)=54.12, p<.001]$. On each day, the mean escape latency with strong shock was significantly longer than with weak shock [Day $1: F(1,60)=35.00$, $\mathrm{p}<.001$; Day 2: $\mathrm{F}(1,58)=4.37, \mathrm{p}<.05$ ]. Neither reward magnitude nor any of the interactions was reliable. The deleterious effect of strong shock on escape latencies is consistent with some previous reports (e.g., McAllister et al., 1971; Moyer \& Korn, 1964). Presumably, responses incompatible with the efficient performance of the escape response were elicited to a greater degree by strong than by weak shock.

\section{Discussion}

Typically, in two-way avoidance learning, weakshock subjects outperform strong-shock subjects from the outset of training (e.g., Bauer, 1972; Dieter, 1977; McAllister et al., 1976; Moyer \& Korn, 1964). Contrary to this usual result, performance was equal for the two shock conditions early in training in the present experiment, a finding consistent with that of Dieter (1977), and was directly related to shock intensity later in training. This modification in the usual relationship between avoidance performance and shock intensity is attributable to the preexposure treatment which presumably reduced the amount of fear condi- 
tioned to situational cues during subsequent avoidance learning. Even with the preexposure treatment, however, some fear does become conditioned to the situational cues early in training when shock is strong, as will be shown in Experiment 2, and presumably, some fear, although less, would also become conditioned with weak shock. The presence of situational-cues fear following the avoidance response is assumed to play a greater negative role in determining effective reinforcement when shock is strong than when weak. Therefore, the reduction in the conditioning of fear to these cues during avoidance learning following preexposure would be expected to increase effective reinforcement more in the strong- than in the weak-shock condition. This greater increase was sufficient in the present experiment to equate the level of effective reinforcement in the two shock conditions, and hence, to equate the level of performance over the first 40 trials. The superiority of performance with strong shock, which emerged later in training, probably depended on the role played by extinction during avoidance learning. As the number of avoidance responses increased with trials, the subjects were exposed to the situational cues in the absence of shock for progressively longer periods of time so that fear of those cues could partially extinguish under both shock conditions. Although extinction could also occur to the CS, the amount should be minimal because of the limited exposure to this stimulus. The decrease through extinction in fear of situational cues present following a response would reduce the negative effect of this source of fear and thereby increase effective reinforcement. Because, theoretically, this increase should be greater for the strong-than for the weakshock condition, performance should eventually become superior with strong shock, as was found.

Reward magnitude, as manipulated in this experiment, should be a relevant variable, according to the effective reinforcement theory, only when some fear of the situational cues is present so that their removal after a response could lead to a decrease in fear. As mentioned above, even with preexposure, fear of the situational cues is present early in training. It is reasonable to assume that, because of the locus of shock, this fear is conditioned primarily to the tactual cues of the grid rather than to the visual cues. Therefore, the delayed occurrence of the reward effect, inasmuch as it is based on fear of visual situational cues, is understandable. Further, because the conditioning of fear to the visual situational cues should progress more rapidly in the strong- than in the weak-shock condition, the reward effect should emerge sooner when shock is strong, as was found.

It should be noted that a negative relationship existed between the escape and avoidance latencies. Although strong shock facilitated avoidance responding, it interfered with escape responding. Nevertheless, at both shock levels, escape latencies decreased over trials, and with strong shock, avoidance latencies decreased over trials on Day 2.
These findings are contrary to the contention of Bolles, Moot, and Nelson (1976) that escape and avoidance latencies are invariant.

A comment should be made about the visual component of the CS used in the two reward conditions. The amount of change in illumination was approximately equal for large and small reward, 1,029 and $1,028 \mathrm{~lx}$, respectively. However, the change was from 28 to $1,056 \mathrm{~lx}$ for small reward and from 0 to $1,0291 \mathrm{x}$ for large reward. Thus, shock was paired with a more intense stimulus in the small-reward condition. If more fear were conditioned to the more intense stimulus (dynamism effect), an advantage would accrue to the small-reward condition and the test of the reward manipulation would be conservative. On the other hand, it is possible that the visual CS was more salient in the large- than in the small-reward condition, despite its weaker absolute intensity, because it involved an increase in illumination from darkness. If, for this reason, more fear were conditioned to this stimulus, an advantage would be given to the large-reward condition and potentially could account for the obtained reward effect. This explanation seems unlikely, however, because, on this basis, the reward effect should have appeared early in training and should not have manifested itself at different stages of training for the two shock conditions. Further, in the McAllister et al. (1976) study, the occurrence of the reward effect with weak but not with strong shock is not explicable in terms of differential fear of the CS, particularly in view of the fact that in the present experiment the reward effect appeared sooner in the strongthan in the weak-shock condition. The reward interpretation provided by the effective reinforcement theory seems to be the most satisfactory explanation of the findings.

\section{EXPERIMENT 2}

The explanation of the results of Experiment 1 was based on the premise that prior exposure to situational cues minimized the amount of fear conditioned to those cues during subsequent avoidance learning. In the present experiment, the amount of fear elicited by the situational cues following a constant amount of avoidance training was directly measured. Ten two-way avoidance training trials were preceded by $4 \mathrm{~h}$ of exposure to the situational cues for one group and not for another. Subsequently, the subjects were allowed to escape, in the absence of shock, from the avoidance apparatus to an adjacent safe compartment. The speed of escaping was taken as an index of the fear which had been conditioned during avoidance training.

\section{Method}

Apparatus. The shuttlebox, with modifications described below, was the one used in Experiment l. An additional feature of the apparatus not used or described in that experiment was 
also employed. Attached at right angles to one outside wall of each of the shuttle compartments was another compartment which was used as a safe box during escape-from-fear training. Each of these safe boxes, $279 \mathrm{~mm}$ long $\times 114 \mathrm{~mm}$ wide $\times 127 \mathrm{~mm}$ high, was painted gray and had a wooden floor hinged to serve as a floor switch. Hinged to the top of each safe box was another box with a translucent plastic bottom which served as a cover and contained a light source to provide constant illumination of $10 \mathrm{~lx}$. These safe boxes could be entered from one of the compartments of the shuttlebox through an opening $63 \mathrm{~mm}$ wide $\times 89 \mathrm{~mm}$ high. Covering this opening was a guillotine door which rested on a hurdle $63 \mathrm{~mm}$ above the floor of the shuttle compartment and $38 \mathrm{~mm}$ above the safe-box floor. The guillotine doors were painted white on the shuttlebox side and gray on the safe-box side. Only one of these safe boxes was used in this experiment.

After the first six subjects in each group were treated, it was necessary, for a purpose unrelated to this experiment, to make some modifications in the shuttlebox apparatus. The dimensions of the compartments remained essentially the same $(231 \times 162$ $\times 152 \mathrm{~mm}$ ), but the guillotine door was increased in width from 57 to $125 \mathrm{~mm}$ and painted white. This difference in apparatus was used as a factor in the statistical analyses and will be referred to as the manipulation of size of door (small vs. large) because that was the only major modification in the apparatus.

For all subjects, the small-reward condition with 1.6-mA shock, as described for Experiment 1, was used in avoidance training. The intertrial illumination was $27 \mathrm{~lx}$ and was increased to $980 \mathrm{~lx}$ with the onset of the visual component of the compound CS. The white-noise component of the CS was an increase in the ambient noise level in the apparatus from 50 to $61 \mathrm{~dB}$.

Subjects and Design. The 96 subjects were of the same age and sex and from the same source as in Experiment 1 . The first 24 subjects were in the small-door condition; the remaining 72 subjects, in the large-door condition. Within each of these conditions, the subjects were assigned randomly in equal numbers ( $n=6$ and 18 ) to one of four experimental groups. Four additional subjects, all in the large-door condition, were discarded, one because of experimenter error and three for meeting a criterion which specified that subjects would be discarded if they locomoted between compartments during the CS-UCS interval on four consecutive trials before the first shock was received. A second discard criterion, failure to escape shock in $60 \mathrm{sec}$ on any trial, was not met by any of the subjects. Except during the experimental treatments, subjects were housed in groups of four with food and water available ad lib.

Half of the subjects were exposed to the situational cues of the shuttlebox prior to avoidance training (preexposure, P) and half were not (no preexposure, NP). Within each preexposure condition, escape-from-fear training, which followed avoidance learning, was administered to half of the subjects with the CS and to half without it (NCS). The groups were designated P-CS, P-NCS, NP-CS, and NP-NCS.

Procedure. On each of the first 4 days of the experiment, the subjects were brought to the experimental room in groups of four, one subject from each group, and were randomly assigned to pairs consisting of one $P$ and one NP subject. On each day, one pair of subjects was handled, as described in Experiment 1, for $\mathbf{2 . 5} \mathrm{min}$ while the other pair remained in the carrying basket, after which the second pair was handled and the first pair was returned to the carrying basket. Then the $P$ subjects were exposed to the situational cues of the apparatus for $1 \mathrm{~h}$, while the NP subjects were placed in neutral holding boxes located adjacent to the shuttlebox. The subjects in the $P$ condition were moved every $15 \mathrm{~min}$ from one shuttle compartment to the other, in the manner described for Experiment 1, and the subjects in the NP condition were similarly moved from one holding box to the other. Thus, at the end of the 4 th day, each subject had received a total of $4 \mathrm{~h}$ of exposure to the situational cues of the apparatus or to the neutral holding boxes. After the $P$ or NP treatment on the 4th day, each subject was placed in the safe box for $2.5 \mathrm{~min}$ while the other subjects were retained in the carrying basket.
On the 5 th day, all subjects received 10 two-way avoidance learning trials. Locomotor responses which occurred before the first shock was received were disregarded. The procedures for each trial were identical to those described for the $1.6-\mathrm{mA}$ smallreward condition of Experiment 1.

On the 6th day, all subjects received 25 escape-from-fear (hurdle-jumping) trials during which no shock was administered. On each trial, the subject was placed in the shuttle compartment, which was adjacent to the safe box employed in this experiment. After $10 \mathrm{sec}$, the guillotine door leading to the safe box was raised. For the subjects in the CS condition, the compound CS used during avoidance training was presented simultaneously with the opening of the door and was terminated by depression of the floor switch in the safe box. After jumping, the subject remained in the safe box for $10 \mathrm{sec}$ and then was removed to a retaining box for a $30-\mathrm{sec}$ ITI. If the subject did not jump within $60 \mathrm{sec}$, the guillotine door was lowered, the subject was immediately removed to the retaining box, and a 60 -sec latency was recorded. Latencies were measured with a Hunter Klockounter in units of $.01 \mathrm{sec}$ from the opening of the guillotine door to the depression of the floor switch in the safe box. The door between the two shuttle compartments remained closed during these trials. Except as specified above, all experimental conditions and procedures were the same as in Experiment 1.

\section{Results}

Avoidance performance. The relationship between the number of avoidance responses and preexposure to situational cues was evaluated with chisquare tests. For the large-door condition, the data were categorized into a 2 by 4 table, $P$ and NP orthogonal to number of avoidance responses $(0$, 1,2 , and $>2$ ). The null hypothesis was rejected $\left[\chi^{2}(3)=8.02, p<.05\right]$, reflecting the greater number of avoidance responses made by the $P$ relative to the NP subjects. In the $P$ condition, 16 of the 36 subjects $(\mathbf{4 4 \% )}$ made two or more avoidance responses compared to 7 of the 36 subjects $(19 \%)$ in the NP condition. Because of the small number of subjects in the small-door condition, the data were categorized into a 2 by 2 table, P and NP orthogonal to number of avoidance responses $(0$ and $>0)$. The null hypothesis was rejected $\left[\chi^{2}(1)=\right.$ $5.04, \mathrm{p}<.05$ ], again reflecting superior avoidance performance for the $P$ relative to the NP subjects. In this case, 6 of the $12 P$ subjects $(50 \%)$ and 1 of the $12 \mathrm{NP}$ subjects $(8 \%)$ made one or more avoidance responses. The findings with the smalldoor condition were corroborated by an identical analysis using data from the first 10 avoidance trials of $\mathbf{P}$ and NP groups from another experiment conducted in this laboratory (Dieter, 1977) with the same apparatus, general procedures, and shock level as used in the present study $\left[\chi^{2}(1)=6.35, p<.02\right]$. In this experiment, one or more avoidance responses were made by 10 of the $16 P$ subjects $(63 \%)$ and by 3 of the 16 NP subjects $(19 \%)$. On the basis of these three analyses, the conclusion is clear: Preexposure to situational cues facilitates two-way avoidance responding.

Escape-from-fear performance. Figure 3 presents the mean hurdle-jumping performance for the 


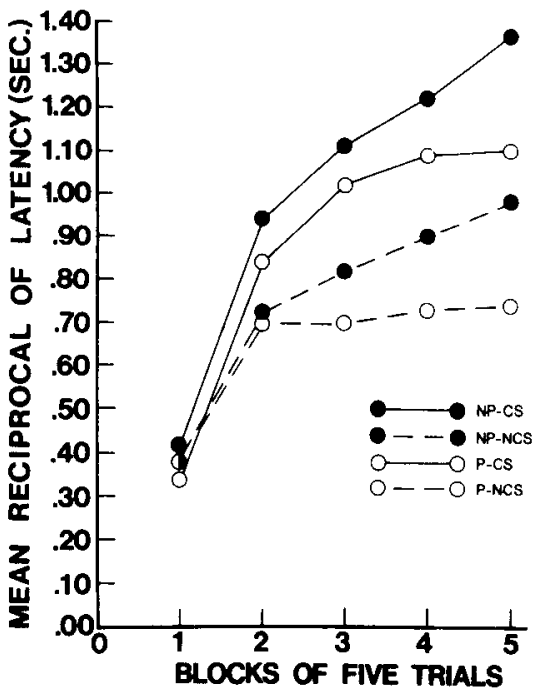

Figure 3. Mean speed of hurdle jumping as a function of blocks of five trials for groups preexposed (P) or not preexposed (NP) to the situational cues prior to two-way avoidance training and given hurdle-jumping, escape-from-fear training in the presence (CS) or absence (NCS) of the diserete CS.

several groups with the size-of-door factor collapsed. Escape from the situational cues alone was slower for the P group (P-NCS) than for the NP group (NP-NCS). Presentation of the CS increased performance of both the $P$ and NP groups to about the same extent.

A repeated-measures ANOVA with preexposure (P vs. NP), stimulus during hurdle jumping (CS vs. NCS), and size of door during avoidance training ( $\mathrm{S}$ vs. L) as the between-subjects factors and blocks of five trials as the within-subject factor yielded significant interactions between trial blocks and preexposure $[F(4,352)=3.10, p<.025]$ and between trial blocks and stimulus during hurdle jumping $[F(4,352)=8.96, p<.001]$. Because of these interactions with trial blocks, terminal performance (Trial Block 5) was analyzed with a factorial ANOVA. Both preexposure and stimulus during hurdle jumping were significant $[F(1,88)=5.50$, $11.57 ; \mathrm{p}<.025,=.001$, respectively]. Neither size of door nor any of the interactions approached significance.

Both the inferior hurdle-jumping performance and the superior avoidance performance of the $P$ relative to the NP condition is attributable to a lesser amount of fear of the situational cues. Presumably, preexposure decreased the conditioning of fear to these cues during avoidance learning (latent inhibition), a circumstance which would lead to the pattern of results obtained. It could be argued, however, that preexposure had a direct facilitatory effect on avoidance performance and that the subsequent poorer hurdle jumping resulted solely from the receipt of fewer shocks in the $\mathrm{P}$ condition. A comparison of the hurdle-jumping performance of sub- jects receiving a constant number of shocks can provide some evidence regarding these two interpretations. To this end, performance on Trial Block 5 of the 67 subjects that had received 9 or 10 shocks was analyzed with a factorial ANOVA (unweighted means) with preexposure (P vs. NP), stimulus during hurdle jumping (CS vs. NCS), and number of shocks as the factors. The effects of preexposure and of stimulus during hurdle jumping were significant $[F(1,59)=7.25,11.21 ; p<.01,<.005$, respectively]. Neither the number-of-shocks factor nor any of the interactions approached significance. The mean speed of hurdle jumping for the $\mathrm{P}$ condition $(.86 \mathrm{sec})$ was significantly lower than that for the NP condition $(1.26 \mathrm{sec})$, indicating that, with a constant number of shocks, preexposure decreased the amount of fear conditioned to the situational cues. Thus, the latent inhibition interpretation of the effects of preexposure is supported. As expected, the CS condition resulted in significantly faster hurdle jumping $(1.32 \mathrm{sec})$ than the NCS condition $(.81 \mathrm{sec})$.

It has been suggested (Bauer, 1972) that escapefrom-fear performance used as a measure of the fear conditioned during avoidance training may be affected by negative transfer. For instance, better twoway avoidance learning might be accompanied by poorer escape-from-fear learning simply because during escape training more of the subjects that avoided well oriented visually and posturally toward the door separating the shuttle compartments than toward the door leading to the safe box. To evaluate this possibility, observations of the orientation of the subjects were made during each trial (i.e., following the opening of the door and, in the appropriate groups, the simultaneous onset of the CS). Of the 96 subjects, 25 showed some orientation, 16 on one, 5 on two, and the remaining 4 on three, four, or six trials, with $77 \%$ of the 43 instances of orientation occurring on Trials 1-4. The tendency to orient was not related to the preexposure variable (12 P vs. $13 \mathrm{NP}$ subjects). However, orientation was significantly related $\left[\chi^{2}(1)=4.84, \mathrm{p}<.05\right]$ to the presence or absence of the CS (18 CS vs. 7 NCS subjects). Despite this relationship, speed of escaping was faster in the CS than in the NCS groups. Thus, the negative transfer that may have occurred was not of sufficient magnitude to obscure the typical beneficial effect of a CS in this task. Furthermore, the pattern of performance remained the same when the data from all subjects that showed orientation were removed. Such a finding is not unexpected, because orientation occurred on only $2 \%$ of the trials.

\section{Discussion}

The interpretation offered for the results obtained in Experiment 1, as well as those of Dieter (1977), is strongly supported by the outcome of the present experiment. That less fear of the situational cues 
was present after avoidance training when subjects had previously been exposed to these cues, as compared to nonpreexposed subjects, was demonstrated by their slower speeds in the escape-from-fear task. This lesser amount of fear can reasonably be considered to be the basis for the superior two-way avoidance performance obtained in the preexposed groups, as would be expected by the effective reinforcement theory.

Two previous studies manipulating preexposure to situational cues have employed a one-way avoidance task with mice (Grant \& Grant, 1973; Grant \& Young, 1971). Because the start and safe boxes of their apparatus were identical, the facilitation which they obtained with preexposure can be explained in the same manner as the similar finding in the twoway task. That is, the lesser amount of fear of situational cues present after the response, when these cues had been preexposed, increased effective reinforcement. Dieter (1978) obtained this same facilitative effect in the one-way avoidance task with rats following preexposure to the start box when the start and safe boxes were highly similar. On the other hand, he found that preexposure to the start box degraded performance when the start and safe boxes were highly dissimilar. In this case, preexposure would interfere with performance because less fear would be conditioned to the situational stimuli of the start box, and hence, less fear reduction would occur following the response.

\section{GENERAL DISCUSSION}

There have been only a few serious attempts to account for the well-established finding that, with standard two-way avoidance procedures, shock intensity and performance are related inversely. One explanation assumes that with increasing shock intensity, there is a concomitant increase in the frequency of competing responses, such as freezing (Bintz, 1971) or "staying" (Theios et al., 1966), which interfere with avoidance responding. This account has previously been found wanting. McAllister et al. (1971) demonstrated that two-way avoidance performance was inferior with strong as compared to weak shock, but that subsequently the strong-shock subjects escaped from the cues of the shuttlebox to an adjoining neutral box faster than did the weak-shock subjects. Neither the CS nor shock was present on these escape-from-fear trials. If the inferior avoidance performance of the strongshock subjects is attributed to the occurrence of competing responses, then such responses should have also interfered with their performance in the escape-from-fear task, but such interference did not occur. The same kind of problem would be faced by a competing-response theory in accounting for the results of Experiment 2. The inferior avoidance performance found in the NP, relative to the $P$, subjects could be attributed to the greater occurrence of competing responses. However, such competing responses should also have led to interference in the escape-from-fear task, but here, the NP subjects showed superior performance. Further evidence against the competing-response hypothesis is that in a one-way avoidance task performance is superior with strong as compared to weak shock (e.g., Dieter, 1976; Moyer \& Korn, 1966). If the competingresponse explanation is used to account for the impairment in performance of the two-way task as a result of strong shock, it should be equally applicable to the one-way task. This rejection of competing responses as an explanation of the inverse relationship does not deny that freezing or crouching responses occur in fear situations. They do, but it is held here that such immobility responses persist only when effective reinforcement for some other response (escaping) is insufficient (McAllister et al., 1971, pp. 431-432). Therefore, the more basic explanation of the inverse relationship is that a shuttle response receives less reinforcement when shock is strong than when it is weak.

Another theory which offers an explanation of the inverse relationship has been provided by D'Amato (D'Amato, Etkin, \& Fazzaro, 1968; D'Amato, Fazzaro, \& Etkin, 1968). This theory holds that avoidance performance depends on two processes: the making of anticipatory responses and the discrimination of the avoidance contingency. Anticipatory responses have been identified in experiments which have shown that responses will occur in the CS-UCS interval even when shock is unavoidable. It is assumed that such anticipatory responses will also occur under usual avoidance learning conditions and, thereby, allow the subject to discriminate the avoidance contingency. Because more anticipatory responses occur with weak than with strong shock, the avoidance contingency is discriminated sooner under the former condition, and the inverse relationship is predicted. However, it is not clear how this explanation can handle the direct relationship obtained in Experiment 1 when the subjects were preexposed to the situational cues. Because shocks were received by all subjects from the outset of training, those with strong shock should have made fewer anticipatory responses and, hence, should have been at a disadvantage, compared to those with weak shock, with respect to discriminating the avoidance contingency. Thus, avoidance performance should have been inferior with strong shock, not superior as was found. To account for the data, this theory needs to incorporate some mechanism whereby preexposure gives an advantage to strong-shock subjects sufficient to overcome the disadvantage engendered by the lesser occurrence of anticipatory responses. It needs also to specify why, at a constant shock level, preexposed subjects perform better than nonpreexposed subjects.

The effective reinforcement theory (McAllister et al., 
1971, 1976), which has been explained above, provides an explanation of the inverse relationship between shock intensity and avoidance performance. It appears to be the only theory which can also account for the direct relationship obtained in Experiment 1.

There are other general theories of avoidance learning which have not attempted to account for the effect of shock intensity in two-way avoidance learning. Bolles $(1970, \mathrm{pp} .40,44)$ has suggested that the learning of an avoidance response depends on the information that shock is not going to occur which is provided the subject by the stimuli consequent to the response (e.g., CS termination). Seligman and Johnston (1973) hold that avoidance learning depends on the acquisition of the expectancy that responding will lead to no shock, the expectancy that not responding will lead to shock, and the fact that no shock is preferred to shock. Herrnstein (1969) assumes that the reinforcement for the learning of an avoidance response is the decrease in the frequency of . aversive stimulation. None of these theories seems to provide a basis for predicting an inverse relationship between shock intensity and avoidance performance in the standard two-way task or for the direct relationship obtained in the present experiment when the situational stimuli were preexposed.

\section{REFERENCES}

ANDERson, N. H. Variation of CS-US interval in long-term avoidance conditioning in the rat with wheel turn and with shuttle tasks. Journal of Comparative and Physiological Psychology, 1969, 68, 100-106.

Anisman, H., \& Waller, T. G. Facilitative and distuptive effects of prior exposure to shock on subsequent avoidance performance. Joumal of Comparative and Physiological Psychology, 1972, 78, 113-122.

BAUER, R. H. The effects of CS and US intensity on shuttlebox avoidance. Psychonomic Science, 1972, 27, 266-268.

Bintz, J. Between- and within-subject effect of shock intensity on avoidance in goldfish (Carassius auratus). Journal of Comparative and Physiological Psychology, 1971, 75, 92-97.

Bolles, R. C. Species-specific defense reactions and avoidance learning. Psychological Review, 1970, 77, 32-48.

Bolles, R. C., Moot, S. A., \& Nelson, K. Note on the invariance of response latency in shuttlebox avoidance learning. Learning and Motivation, 1976, 7, 108-116.

Camprele, B. A. The reinforcement difference limen (RDL) function for shock reduction. Journal of Experimental Psychology, 1956, 52, 258-262.

Campbell, B. A., \& Kraeling, D. Response strength as a function of drive level and amount of drive reduction. Journal of Experimental Psychology, 1953, 45, 97-101.

Cicala, G. A., \& Kremer, E. The effects of shock intensity and d-amphetamine on avoidance learning. Psychonomic Science, 1969, 14, 41-42.

D'Amato, M. R., Etkin, M., \& Fazzaro, J. Effects of shock type and intensity on anticipatory responses. Journal of Comparative and Physiological Psychology, 1968, 66, 527-529.

D'Amato, M. R., \& Fazzaro, J. Discriminated lever-press avoidance learning as a function of type and intensity of shock. Journal of Comparative and Physiological Psychology, 1966, 61, 313-315.
D'Amato, M. R., Fazzaro, J., \& Etkin, M. Anticipatory responding and avoidance discrimination as factors in avoidance conditioning. Journal of Exprimental Psychology, 1968, 77, 41-47.

Dieter, S. E. Continuity and intensity of shock in one-way avoidance learning in the rat. Animal Learning \& Behavior, 1976, 4, 303-307.

Dreter, S. E. Preexposure to situational cues and shock intensity in two-way avoidance learning. Animal Learning \& Behavior, 1977, 5, 403-406.

Dieter, S. E. Preexposure, exploration, and similarity of the start and safe boxes in one-way avoidance learning. Unpublished doctoral dissertation, Northern Illinois University, 1978.

Freedman, P. E., Hennessy, J. W., \& Groner, D. Effects of varying active/passive shock levels in shuttle box avoidance in rats. Journal of Comparative and Physiological Psychology, $1974,86,79-84$.

Grant, M. J., \& Grant, R. M. The effects of scopolamine on preexposure to a learning apparatus. Bulletin of the Psychonomic Society, 1973, 1, 238-240.

Grant, M., \& Young, D. The effects of preexposure to a learning apparatus. Behavior Research Methods \& Instrumentation, 1971, 3, 251-252.

Herrnstein, R. J. Method and theory in the study of avoidance. Psychological Review, 1969, 76, 49-69.

Kurtz, P. S., \& Shafer, J. N. The interaction of UCS intensity and intertrial interval in avoidance learning. Psychonomic Science, 1967, 8, 465-466.

LEVINE, S. UCS intensity and avoidance learning. Journal of Experimental Psychology, 1966, 71, 163-164.

Lubow, R. E. Latent inhibition. Psychological Bulletin, 1973, 79, 398-407.

McAllister, D. E., McAllister, W. R., \& Dieter, S. E. Reward magnitude and shock variables (continuity and intensity) in shuttlebox-avoidance learning. Animal Learning \& Behavior, 1976, 4, 204-209.

McAllister, W. R., McAllister, D. E., \& Douglass, W. K. The inverse relationship between shock intensity and shuttle-box avoidance learning in rats: A reinforcement explanation. Journal of Comparative and Physiological Psychology, 1971, 74, 426-433.

MODARESI, H. A. One-way characteristic performance of rats under two-way signaled avoidance conditions. Learning and Motivation, 1975, 6, 484-497.

Mowrer, O. H. On the dual nature of learning-A re-interpretation of "conditioning" and "problem-solving." Harvard Educational Review, 1947, 17, 102-148.

MOYER, K. E., \& KORN, J. H. Effect of UCS intensity on the acquisition and extinction of an avoidance response. Journal of Experimental Psychology, 1964, 67, 352-359.

MOYER, K. E., \& KORN, J. H. Effect of UCS intensity on the acquisition and extinction of a one-way avoidance response. $P_{s y}$ chonomic Science, 1966, 4, 121-122.

Rescorla, R. A. Summation and retardation tests of latent in hibition. Joumal of Comparative and Physiological Psychology, 1971, 75, 77-81.

Seligman, M. E. P., \& Johnston, J. C. A cognitive theory of avoidance learning. In F. J. McGuigan \& D. B. Lumsden (Eds.), Contemporary approaches to conditioning and learning. Washington, D.C: Winston, 1973.

Theios, J., Lynch, A. D., \& Lowe, W. F., JR. Differential effects of shock intensity on one-way and shuttle avoidance conditioning. Joumal of Experimental Psychology, 1966, 72, 294-299.

(Received for publication March 31, 1978; revision accepted August 22, 1978.) 